\title{
Learning from the proteasome how to fine-tune cancer
}

\section{immunotherapy}

Nathalie Vigneron ${ }^{1,2, *}$, Joanna Abi-Habib ${ }^{1,2}$, Benoit J. Van den Eynde ${ }^{1,2,3}$

${ }^{1}$ Ludwig Institute for Cancer Research, Brussels, Belgium

${ }^{2}$ de Duve Institute, Université catholique de Louvain, Brussels, Belgium

${ }^{3}$ WELBIO (Walloon Excellence in Life Sciences and Biotechnology), Brussels, Belgium

* Author to whom correspondence should be addressed: Dr. Nathalie Vigneron, Ludwig Institute for Cancer Research, de Duve Institute, Université catholique de Louvain, Avenue Hippocrate 75 B1.74.03, B-1200 Brussels, Belgium; Tel.: +32-2-7647558; Fax: +32-2-7647590; E-Mail: nathalie.vigneron@bru.licr.org.

Keywords: Immunotherapy, cytolytic T lymphocytes, MHC class I, proteasome, tumor antigens

\begin{abstract}
Cancer immunotherapy recently emerged as a forefront strategy to fight cancer. Key players of the anti-tumor responses are $\mathrm{CD} 8^{+}$cytolytic $\mathrm{T}$ lymphocytes (CTL), which can detect tumor cells that carry antigens, i.e. small peptides bound to surface HLA class I molecules. The success and safety of cancer immunotherapy strategies depends on the nature of the antigens recognized by the targeted $\mathrm{T}$ cells, their strict tumor specificity and whether tumors and antigen-presenting cells can efficiently process the peptide. Here, we
\end{abstract}


review the nature of the tumor antigens and their potential for the development of immunotherapeutic strategies. We also discuss the importance of proteasome in the production of these peptides in the context of immunotherapy and therapeutic cancer vaccines.

\section{1. $\mathrm{CD8}^{+} \mathrm{T}$ cells and cancer immunotherapy}

The notion that the immune system could be exploited to fight cancer emerged decades ago, when it became clear that the immune system, and more specifically $\mathrm{T}$ lymphocytes, were able to detect and eliminate malignant cancer cells. This process, called immune surveillance, partly relies on a specific $\mathrm{T}$ cell subset, the $\mathrm{CD} 8^{+}$cytolytic $\mathrm{T}$ lymphocytes (CTL), whose function is to recognize and kill tumors or virally infected cells. CTL recognize peptides of 8-10 amino acids, which are presented at the cell surface by human leukocyte antigens (HLA) class I molecules and originate from the degradation of intracellular proteins (reviewed in [1]). In order to achieve optimal immune surveillance, cells display a large array of peptides, sampling a variety of proteins expressed. In healthy cells, those peptides originate from normal autologous genes and are ignored by the immune system as a result of self-tolerance. However, after viral infection or cell transformation, this repertoire also includes peptides derived from viral or tumorassociated proteins.

Aimed at boosting the patient's immune response against his tumor, cancer immunotherapy emerges as a new therapeutic approach leading to clinically meaningful regression of metastatic tumors. Adoptive T-cell therapies (see glossary) and monoclonal antibodies blocking the CTLA-4 and PD1 immune checkpoints have induced long-term 
tumor regressions and are becoming key elements of the anti-cancer clinical armamentarium [2, 3]. However, although these therapies induce sustained clinical responses in a large fraction of cancer patients, they also can induce harmful autoimmune side effects $[4,5]$. The design of safer and more efficient immunotherapeutic approaches requires a strict characterization of the targeted antigenic peptides as well as the processes involved in their presentation to the immune system. In this review, we will discuss the nature of the peptides recognized by $\mathrm{T}$ lymphocytes on tumors and the parameters that are involved in their presentation to the immune system.

\section{Human tumor antigens}

A number of peptides recognized by CTLs on tumors were identified ${ }^{i}[6]$. These peptides $^{2}$ are commonly classified according to the expression pattern of the gene from which they derive: antigens with high tumor specificity and those with low tumor specificity (Figure 1) $[2,3]$.

\subsection{Antigens with high tumor specificity}

\subsubsection{Viral antigens}

Viruses have been associated to the development of several types of cancers [7]. Because peptides derived from viral proteins are part of the peptide repertoire of virally induced tumors, these peptides are relevant targets for the development of immunotherapeutic approaches. This is especially true, as T cell precursors recognizing viral antigens are not subjected to negative selection in the thymus (a process required to eliminate $\mathrm{T}$ cells reactive against self-antigens, and which will be discussed in Text Box 1): CTL of high affinity for these antigens should therefore be present in the blood and could potentially 
be activated by immunotherapy regimens. Vaccination with long human-papilloma virus (HPV) peptides emerged as a promising therapeutic modality for HPV-related cancers, as these long peptides proved capable of eliciting HPV-16-specific T cells in patients $[8,9]$. However, whereas long-peptide vaccines prevented disease progression in some patients with premalignant disease [9], it was not the case in patients with advanced or recurrent cervical cancer [10]. Recently, these peptides were administrated in combination with a chemotherapy regimen: this decreased the amount of circulating myeloid-derived suppressor cells (see glossary) and induced strong HPV-specific T cell responses [11].

\subsubsection{Mutated antigens}

Genome instability and mutations are a hallmark of cancer and give rise to mutated antigens, also called neoantigens. Because mutated antigens are not expressed in the thymus, $\mathrm{T}$ cells that recognize them are not deleted in the course of negative selection (Text Box 1), which probably explains the prevalence of the spontaneous anti-tumor responses to mutated antigens in mice and humans [12]. Point mutations produce new antigenic peptides resulting either from a change of one amino acid in the peptide sequence, from the extension of an open-reading frame due to the loss of a stop codon, or from a shift of the reading frame. Interestingly, immune-checkpoint inhibitors are especially efficient against cancers such as melanoma or non-small lung carcinoma, which show the highest rate of somatic mutations, or against cancers that bear a deficiency in DNA mismatch repair mechanisms and show high mutation load [13-15]. Moreover, acquired resistance to immune-checkpoint inhibitors was shown to involve the loss of neoantigens recognized by circulating $\mathrm{T}$ cells, suggesting that these neoantigens could have been the target of anti-tumor responses induced by immune-checkpoint 
inhibitors [16]. Consequently, targeting mutated antigens could be an efficient means to fight cancer. In the past, mutated antigens were often disregarded in the context of vaccine development; first because their identification was often challenging and second because mutated antigens are unique to an individual patient and therefore not exploitable for generalized immunotherapeutic approaches. However, the recent improvement of personalized high-throughput whole-exome sequencing (WES) has opened new opportunities for the use of neoepitopes. A large series of peptides derived from mutations and recognized by autologous tumor-infiltrating lymphocytes (TIL) were identified using WES coupled to HLA class I binding algorithms, HLA-peptide tetramer libraries and/or minigene libraries [17-20]. Combining WES and direct mass spectrometry identification of peptides eluted from the surface of tumors also led to the identification of novel neoepitopes recognized by autologous TIL [21]. The direct transfer of unmodified TIL recognizing neoantigens showed durable complete tumor regression of multiple metastases in some patients [18, 22]. Surprisingly, in HPV-induced epithelial cancers, immunodominant (see glossary) $\mathrm{T}$ cell reactivity occurs against mutated antigens or a MAGE-type antigen (see below) rather than against HPV-derived antigens [23].

\subsubsection{Antigens encoded by cancer-germline genes}

Cancer-germline genes are expressed in a wide variety of cancer types, but not in healthy tissues except germline and trophoblastic cells (reviewed in [2, 3]). The prototypes of cancer-germline genes are the genes of the melanoma-antigen encoding gene (MAGE) family (reviewed in $[2,3]$ ), whose specific expression in tumors has been linked to the demethylation of their promoter sequence, as part of a genome-wide demethylation (see 
glossary) that takes place in male germline cells and in some advanced cancers (reviewed in $[2,3])$. However, because male germline cells do not express surface MHC class I molecules, the peptides derived from cancer-germline genes are not expressed on these cells. Antigens encoded by cancer-germline genes are known as MAGE-type antigens. They are made of a peptide derived from the MAGE protein and presented by a given HLA class I molecule. As opposed to mutated antigens, they are shared by many tumors. This feature combined to their strict tumor-specific expression makes MAGE-type antigens attractive targets for cancer immunotherapy.

\subsection{Antigens with low tumor specificity}

\subsubsection{Differentiation antigens}

Differentiation antigens are derived from genes that are expressed in specific healthy tissues as well as in the corresponding tumor. Most differentiation antigens identified to date derive from proteins involved in the biogenesis of melanosomes or melanin synthesis such as tyrosinase, gp100 or TRP1 (reviewed in $[2,3]$ ). T cell responses to melanoma differentiation antigens can lead to vitiligo, a partial skin depigmentation, which is often observed in melanoma patients and is generally associated with a good prognosis [24]. Another example of differentiation antigen is the carcinoembryonic antigen (CEA), which is often highly expressed in colorectal cancer and some other carcinomas, but is also found at lower level in a variety of normal epithelial cells of the intestinal tract (reviewed in [25]). 


\subsubsection{Overexpressed antigens}

Overexpressed antigens are produced from genes that are highly expressed in tumors and show a lower but detectable expression in healthy tissues. Examples of overexpressed antigens from which antigenic peptides recognized by CTLs were identified are PRAME HER2/NEU or MOK (RAGE-1), (reviewed in $[2,3]$ ). The risk of using such antigens remains uncertain, as they are expressed in normal tissues at low but still significant levels. Defining the threshold of expression above which an antigenic peptide will be detected by the immune system remains a critical issue that is difficult to address and renders the use of these antigens quite hazardous.

\section{Processing of tumor antigens}

The production of antigenic peptides recognized by CTL is a continuous process that starts in the cytoplasm, with the degradation of cellular proteins by the proteasome (Figure 1). Peptides released in the cytosol following proteasome degradation are then transported in the lumen of the endoplasmic reticulum (ER) by a dedicated transporter, called transporter associated with antigen processing (TAP). Once in the ER lumen, peptides can be further trimmed by ER-associated aminopeptidases (see glossary) ERAP1 or ERAP2, and peptides of the appropriate size (about 8-10 amino acids) are then loaded on major histocompatibility complex (MHC) class I molecules with the help of the peptide loading complex, which is composed of TAP, tapasin, ERp57 and calreticulin. Peptide/MHC complexes then exit the ER, follow the secretory pathway and reach the cell surface where they can be recognized by CTLs. 


\subsection{The proteasome and antigen presentation}

The proteasome is a component of the ubiquitin-proteasome system, which is the major ATP-dependent proteolytic machinery in cells. It is responsible for the bulk of protein degradation and it is therefore crucial to the maintenance of cell homeostasis, as well as cell differentiation, cell-cycle progression or apoptosis. In the course of protein degradation, the proteasome releases peptides that are then loaded on HLA class I and constitute the peptide repertoire detectable by the immune system (Text Box 2).

\subsubsection{The proteasome}

The 20S proteasome is a barrel-shaped structure composed of four stacked heptameric rings that delimit a catalytic chamber inside which proteins are degraded (Figure 2). The two outer rings are made of $\alpha$ subunits ( $\alpha 1-7)$ while the two inner rings are made of $\beta$ subunits $(\beta 1-7)$, three of which $(\beta 1, \beta 2$, and $\beta 5)$ are catalytically active in vertebrate proteasomes. Entrance of proteins into the catalytic chamber of the proteasome is tightly regulated, to protect cells from detrimental protein degradation: a very narrow pore delimited by the $\alpha$-subunits is further obstructed by the $N$-terminal tail of the $\alpha$-subunits, which restrain the entry of folded protein substrates inside the catalytic chamber [26]. Opening of the $\alpha$-gate is regulated through the binding of regulatory particles, which interact with the $\mathrm{N}$-terminal tails of the $\alpha$-subunits to open the gates [27]. Four types of proteasome regulators exist (Figure 2): the 19S regulator (PA700), the 11S regulators PA28 $\alpha \beta$ and PA28 $\gamma$, and the PA200 regulator (reviewed in [28]). Although an additional particle, PI31 was suggested to bind proteasomes [29], its effective contribution to proteasome function is still a matter of debate [30, 31]. Degradation of folded, polyubiquitinated proteins requires the $19 \mathrm{~S}$ regulatory particle, which binds, unfolds, 
deubiquitinates and translocates proteins inside the catalytic chamber of the proteasome [28]. The nature of the regulator associated to the $20 \mathrm{~S}$ proteasome can influence the proteasome ability to release some specific antigenic peptides [32,33].

\subsubsection{Proteasome catalytic activity and peptide splicing}

Mutagenesis studies as well as structural analyses of proteasomes bound to inhibitors have shown that it is the hydroxyl group of the threonine located at the N-terminal position of the catalytic subunit that initiates peptide bond hydrolysis (Figure 3a) [34, 35]. Three types of $\beta$ subunits are catalytically active in the vertebrate proteasome: $\beta 1$, $\beta 2$, and $\beta 5$. Three major types of activities exist: the caspase-like activity (cleaving after acidic amino acids), the trypsin-like activity (cleaving after positively charged amino acids) and the chymotrypsin-like activity (cleaving after hydrophobic amino acids). Based on the study of yeast proteasome mutants, each of these activities were attributed to a specific type of subunit (reviewed in [28]): the caspase-like activity is linked to the $\beta 1$ subunit, the trypsin-like activity to $\beta 2$ and the chymotrypsin-like activity is associated to the $\beta 5$ subunit. However, some subunits show overlapping specificities, and the amino acids surrounding the cleavage site also influence the cleavage specificity. It is the nature of the amino acids that are lining the structural pockets around the active site, as well the size and structure of these pockets that dictate the cleavage characteristic of each subunit [36].

A few years ago, we have identified a peptide recognized by a CTL on melanoma cells and derived from the splicing of two peptide fragments originally distant in the protein [37]. This phenomenon, called peptide splicing, takes place inside the catalytic chamber 
of the proteasome and involves the creation of a new peptide bond between two distant peptide fragments (Figure 3b). Thus far, six human antigens produced by peptide splicing and recognized by CTL were identified [37-43]. Interestingly, among the 6 peptides described to date four were produced by splicing of peptide fragments in the reverse order to that in which they occur in the parental protein [39, 41-43]. Studying the mechanism of peptide splicing, we showed that peptide splicing occurs by transpeptidation and involves the nucleophilic attack of the acyl-enzyme intermediate by the N-terminus of another peptide fragment present in the chamber [37] (Figure 3b). We also showed that splicing requires the nucleophilic peptide to be at least 3 amino-acid long [42]. Even though condensation has been proposed as an alternative peptide splicing mechanism for at least one peptide, the experimental evidence is limited to results obtained with in vitro digests or with cells expressing appropriately truncated constructs, but not with cells expressing full-length proteins [43]. Moreover, the prominent role of direct transpeptidation over alternative condensation was confirmed by mass spectrometry analysis of peptide digests performed in the presence of isotopically-labeled water [44]. Because peptide splicing potentially involves any peptide fragment released through proteolysis, it could give rise to a large variety of peptides. Until recently, the identification of spliced antigenic peptides has been limited by the availability of specific CTL directed against these peptides. Identification of spliced peptides using mass spectrometry approaches was limited by the fact that peptide databases were only designed to include linear protein fragments. Recently, Liepe et al. circumvented this issue by creating a custom peptide database, which included any possible short peptide created by the splicing of non-contiguous peptide fragments from the proteins expressed 
in the cell lines studied [45]. Analyzing the HLA-I immunopeptidome (see glossary) obtained from different human cell lines, they showed that spliced peptides represented up to $25 \%$ of the total amount of peptides found at the cell surface. This demonstrates that peptide splicing is not a rare event and contributes significantly to the diversity of the peptide repertoire (see glossary).

\subsubsection{Proteasome subtypes}

\subsubsection{Standard and immunoproteasome}

Immune cells and cells treated with inflammatory cytokines, such as IFN $\gamma$, express alternative proteasome catalytic subunits LMP2( $\beta 1 \mathrm{i})$, MECL-1( $(\beta 2 \mathrm{i})$ and LMP7( $\beta 5 \mathrm{i})$, which are incorporated into proteasome instead of $\beta 1, \beta 2$, and $\beta 5$, to form an alternative proteasome subtype, called the immunoproteasome (Figure 2). Because standard proteasomes and immunoproteasomes bear different catalytic subunits, their cleavage specificities are different, as observed by their activity on fluorogenic peptides: the immunoproteasome displays a much lower caspase-like activity while its trypsin-like and chymotrypsin-like activities are increased $[46,47]$. This can be explained by subtle conformational changes in the substrate-binding pockets of catalytic subunits $\beta 1 \mathrm{i}$ and $\beta 5 \mathrm{i}$ [36]: the S1 substrate-binding pocket (which interacts with the amino acid residue forming the C-terminal end of the cleaved peptide) of $\beta 1 \mathrm{i}$ is smaller, more hydrophobic, and lacks a positively charged residue (R45) when compared to that of $\beta 1$, thereby accounting for the reduced caspase-like activity of $\beta 1 \mathrm{i}$ and its higher cleavage activity after small hydrophobic and branched residues [36]. Compared to $\beta 5, \beta 5 \mathrm{i}$ harbors a more spacious S1 pocket favoring the cleavage of large non polar residues like tyrosine, 
tryptophan or phenylalanine [36]. In addition, subunit activity was also suggested to be allosterically regulated by the presence of the other subunits: for example, subunit $\beta 5 i$, by impacting the structure of the 20S proteasome, seems to increase the activity of subunits $\beta 2 \mathrm{i}$ and $\beta 1 \mathrm{i}[48]$.

As HLA class I binding generally calls for peptides with hydrophobic or basic Ctermini, it has been suggested that the immunoproteasome, thanks to its increased chymotrypsin-like and trypsin-like activities, produces peptides that are more likely to bind HLA-molecules with high affinity $[49,50]$. This idea was corroborated by the study of mice knockout for all three immunosubunits in which presentation of a number of MHC class I epitopes was decreased [51]. Moreover, the peptide repertoire of $\beta 2 \mathrm{i}^{-/-} \beta 5 \mathrm{i}^{-/}$ or triple knockout mice cells is different than that of wild type mice [51, 52]. Although overall, immunoproteasomes seem to play an important role in antigen presentation, some peptides expressed on tumors are processed more efficiently by the standard proteasome than the immunoproteasome $[46,53,54]$. The ability of a proteasome subtype to produce an antigenic peptide results from a balance between its ability to produce the cleavages releasing the peptide and its propensity to destroy the peptide by cleaving inside the peptide sequence [55]. Recently, Tripathi et al. observed that lung cancer cells that have undergone epithelial-to-mesenchymal cell transition showed a reduced expression of the immunoproteasome [56]. This was associated with a marked reduction in the diversity of the peptides presented by HLA class I, and a poor clinical outcome in non-small lung carcinoma patients [56]. Interestingly, immunofluorescence studies performed on metastases of three melanoma patients showed a drastic correlation between T-cell infiltration into tumors and the presence of immunoproteasome subunits, 
suggesting that the local production of IFN $\gamma$ by infiltrating lymphocytes might induce the expression of immunoproteasome in surrounding tumor cells [57]. This was also observed in breast cancer, where expression of immunoproteasome genes was correlated with expression of IFN $\gamma$, with the presence of a $\mathrm{T}$ cell infiltrate and with increased survival [58].

\subsubsection{Intermediate proteasomes}

A few years ago, our group identified two additional types of proteasome, which are intermediate between the standard proteasome and the immunoproteasome in that they contain only one ( $\beta 5 \mathrm{i})$ or two $(\beta 1 \mathrm{i}$ and $\beta 5 \mathrm{i})$ of the three catalytic subunits of the immunoproteasome [46] (Figure 2). The existence of such intermediate proteasomes was previously suggested by the fact that some tissues only express some of the three immunoproteasome subunits [59]. It is the use of antibodies able to recognize specifically each immunoproteasome subunit under its native conformation that enabled us to define the exact stoichiometry of intermediate proteasomes and the amount of each proteasome subtype found in a large variety of cell types: intermediate proteasomes $\beta 5 \mathrm{i}$ and $\beta 1 \mathrm{i} \beta 5 \mathrm{i}$ represent 10 to $20 \%$ of the total proteasomes found in tumors and 30 to $50 \%$ of those found in liver, kidney, small bowel, colon and dendritic cells [46]. Studying the catalytic activity of intermediate proteasomes in native conditions, we showed that intermediate proteasome $\beta 5$ i displayed a chymotrypsin-like activity that is intermediate between the standard and the immunoproteasome, while that of $\beta 1 \mathrm{i} \beta 5 \mathrm{i}$ is similar to the immunoproteasome $[46,47]$. The trypsin-like activities of both $\beta 5 \mathrm{i}$ and $\beta 1 \mathrm{i} \beta 5 \mathrm{i}$ are 
intermediate between the standard and the immunoproteasome. Finally, intermediate proteasome $\beta 5$ i displays a caspase-like (cleavage after acid amino acids) activity similar to that of the standard proteasome, while this activity is low in $\beta 1$ i $\beta 5$ i proteasome. This is because the caspase-like activity is assigned to the $\beta 1$ subunit, which is present in standard and intermediate proteasome $\beta 5 \mathrm{i}$ and absent in the immunoproteasome and intermediate proteasome $\beta 1 \mathrm{i} \beta 5 \mathrm{i}$. Because of their particular cleavage properties, intermediate proteasomes were shown to produce a unique repertoire of peptides $[46,54]$ (Table 1). Hence, intermediate proteasomes enlarge the HLA class I repertoire to peptides that can be processed by both dendritic cells (containing immunoproteasomes and intermediate proteasomes) and tumor cells (containing standard proteasomes and intermediate proteasomes). Such peptides might therefore represent valuable targets for cancer immunotherapy.

\subsubsection{Thymoproteasome}

Finally, the thymic cortex expresses a specific subtype of proteasome, called the thymoproteasome, which comprises $\beta 1 \mathrm{i} \beta 2 \mathrm{i}$ subunits assembled to $\beta 5 \mathrm{t}$, which is a homologue of $\beta 5 / \beta 5 \mathrm{i}$, and is specifically expressed in the cortical thymic epithelial cells (cTEC) (Figure 2)[60]. Because a lack of $\beta 5$ t dramatically alters splenic CTL diversity and mice ability to respond to viral infection [60,61], the thymoproteasome was suggested to be involved in the positive selection of thymocytes (Text Box 1), a process which is mediated by cTEC and triggers the survival of only those T-cell precursors that are able to bind to self-MHC class I/peptide complexes. The exact role played by the thymoproteasome in this process remains to be defined. 


\section{Cancer immunotherapy: Future perspectives}

The rationale of cancer immunotherapy is to activate the immune system so that it can recognize and destroy tumors. Nowadays, three main approaches are followed:

- The injection of antibodies blocking immune-checkpoints (e.g. CTLA-4 or PD1), to boost or reawaken $\mathrm{T}$ cells, regardless of their antigen specificity

- The adoptive transfer of anti-tumor T cells (isolated directly from the patient's tumor or engineered to express a native or affinity-enhanced TCR) or CAR-T cells (engineered to express a chimeric receptor recognizing a surface tumor antigen).

- Therapeutic vaccination, which aims at activating specific anti-tumor $\mathrm{T}$ cells present in the patient's blood, in order to specifically boost the natural anti-tumor immune response.

Although T cell-stimulating antibodies have shown long-term clinical efficacy in a large number of patients, strong autoimmune side effects are often observed $[4,5]$. This is because these antibodies activate all $\mathrm{T}$ cells, regardless of their antigen specificity. Peripheral tolerance to self antigens is released, resulting in autoimmune damage to healthy tissues. The adoptive transfer of in vitro expanded T cells, also gave good clinical results $[62,63]$. However, some trials had to be prematurely closed due to the occurrence of serious, sometimes fatal, outcomes. These deleterious side effects were always related to the use of $\mathrm{T}$ cells expressing $\mathrm{T}$ cell receptors whose affinity had been artificially increased to improve the anti-tumor responses (Text Box 3) [64-68]. In contrast, adoptive transfer of naturally occurring TIL from patients with melanoma, generally showed clinical efficacy and no signs of harmful side effects $[63,69]$. Therefore, caution should 
be taken when using T cells expressing affinity-enhanced TCR, which have bypassed the natural mechanisms of negative selection in the thymus (Text Box 1 and 3). The transfusion of large amounts of $\mathrm{T}$ cells expressing unmodified $\mathrm{T}$ cell receptors and targeting tumor-specific antigens might be an efficient way to fight cancer, but remains difficult to apply to large cohorts of patients. Instead, therapeutic vaccination appears as a more widely applicable strategy to specifically activate anti-tumor T cells. Moreover, because the $\mathrm{T}$ cells targeted by the vaccine went through thymic selection, the occurrence of undesirable immune responses against self-antigens expressed by normal tissues should be minimized. Unfortunately, so far, therapeutic vaccination applied to cancer patients has shown little success with a response rate of only 5 to $10 \%$ [2]. However, a recent long-term follow-up analysis of metastatic melanoma patients having received dendritic-cell based vaccines indicated that their overall survival after 12 years (19\%) was similar to the survival observed with anti-CTLA-4 in similar cohorts of patients [70]. The efficacy of cancer vaccines might largely depend on the mode of delivery of the antigen. In that sense, peptide or protein-based vaccines are not optimal to elicit strong CTL responses. On the contrary, viral vector-based vaccines designed to express antigens of interest are very immunogenic, and were found to induce strong CTL responses, coupled to anti-tumor responses [71]. Moreover, viral vectors can efficiently infect dendritic cells, which are responsible for the priming of naïve anti-tumor $\mathrm{T}$ cells. To limit the risk of tumor escape by selection of antigen-loss variants, cancer vaccines can be designed to activate $\mathrm{T}$ cells against a variety of antigens. In addition, one can favor vaccines targeting antigens that have a high tumor specificity and can be presented by tumors in any circumstances. In that regard, tumor antigens processed by both 
intermediate proteasomes and immunoproteasomes appear as the best choice, since intermediate proteasomes are found in most tumors at steady state while the immunoproteasome is prominent only in tumors exposed to an inflammatory environment [46]. Since dendritic cells express both the intermediate and the immunoproteasome, virus-based vaccines will prime $\mathrm{T}$ cells recognizing antigens processed by these proteasome types (Figure 4). However, vaccine based on full-length viral constructs will be inefficient to induce $\mathrm{T}$ cells against peptides that are better produced by the standard proteasome, such as melanoma differentiation antigens (Table 1) [72]. In those cases, vaccines based on short peptides or minigene constructs (see glossary), which bypass processing in dendritic cells, will be more efficient [72]. In line with this, Dannull et al. showed that the infusion in melanoma patients of mature dendritic cells transfected with RNA encoding full-length tumor antigens, induced stronger anti-tumor CTL responses when these DC were concomitantly transfected with siRNA downregulating the immunosubunits of proteasome [73]. This is likely because DC then express the standard proteasome, which is the main proteasome type present at steady state in tumors. It would be interesting to see whether similar results would be obtained by using specific inhibitors of immunoproteasome subunits, such as ONX 0914, which targets $\beta 5 \mathrm{i}$ [74] or UK-101 and IPSI-001, which target $\beta 1 \mathrm{i}[75,76]$. Thus, the precise knowledge of the type of proteasome involved in the production of an antigenic peptide is essential, to determine the most adequate peptide to target, but also the mode of delivery of the antigen in the context of immunotherapeutic cancer vaccines. In that context, proteasome cleavage prediction algorithms are available, which were generally trained either on in vitro proteasome digestion datasets or on described MHC class I 
ligands (whose C-terminus reflects the proteasome cleavage site) [77]. However, the accuracy of these prediction algorithms remains unclear because the in vivo proteolytic activity generating MHC class I ligands can be very different from the in vitro proteasome proteolysis, given that additional proteases, different proteasome subtypes and their regulators, TAP transport and MHC binding are also involved in the processing of antigenic peptides [77]. The availability of reliable prediction algorithms would be very useful, in particular to predict the processing of mutated antigens, which are nowadays suggested by sequencing of patients' tumor DNA in order to develop personalized cancer vaccines.

Vaccination of cancer patients sometimes leads to the priming of anti-tumor $\mathrm{T}$ cells that target antigens different from those of the vaccine. This phenomenon, called antigen spreading, is likely triggered by the release of antigens from dying tumor cells. After processing by dendritic cells, these antigens will activate $\mathrm{T}$ cells with new specificities [78]. Such priming by dendritic cells favors the activation of $\mathrm{T}$ cells that recognize antigens produced by the immunoproteasome or intermediate proteasomes [78].

Proteasome inhibitors such as bortezomib or carfilzomib have now been approved for the treatment of multiple myeloma. The unique sensitivity of multiple myeloma to these inhibitors is related to their secretion of large amounts immunoglobulins, which makes them highly dependent on the ER-associated protein degradation (ERAD) pathway [79]. This pathway ensures proteasome-mediated degradation of misfolded secretory proteins. By blocking ERAD, proteasome inhibitors induce ER stress leading to the death of myeloma cells. Although their impact on antigen presentation by cancer cells in vivo has 
not been investigated so far, such inhibitors are expected to reduce presentation of tumor antigens. Therefore, caution should be taken when combining proteasome inhibitors with immunotherapy.

\section{Concluding Remarks}

Cancer immunotherapy has now become a prime strategy to fight cancer, due to the development of antibodies targeting immune checkpoints inhibitors. Improvements are still needed, however, to increase the number of patients responding to these treatments and decrease the occurrence of harmful side effects. Many of the non-responder patients have "cold" tumors, which are not infiltrated by $\mathrm{T}$ cells, due in large part to their lack of anti-tumor immune response. Such patients might benefit from cancer vaccines or adoptive $\mathrm{T}$ cell therapies able to trigger anti-tumor immune responses that could then be further boosted by the use of immune checkpoint inhibitors. By triggering tumor-specific $\mathrm{T}$ cell responses, such combinations could also reduce the autoimmune toxicity of immunotherapy. Key considerations for the design of safe and effective immunotherapeutic strategies include the identification of the most specific tumor antigen together with a better characterization of the processing of these peptides, which will guide the choice of the most appropriate vaccine platform (see Outstanding Questions). In order to minimize tumor escape by antigen loss, cancer vaccines should ideally target a variety of distinct antigens. Tumoral immune resistance might result from the expression

by tumors of immunomodulatory molecules that dampen the immune response and inhibit the function of anti-tumor CTL. One example is the tryptophan-degrading enzyme indoleamine 2,3-dioxygenase (IDO), which, by depleting tryptophan and releasing 
tryptophan metabolites, inhibits $\mathrm{T}$ cell proliferation and function while promoting tumor survival and motility [80]. Another example is the programmed death ligand-1 (PD-L1), whose expression on tumor cells is stimulated by IFN $\gamma$ and whose engagement with its $\mathrm{T}$ cell receptor PD-1, leads to a decrease in $\mathrm{T}$ cell proliferation, cytokine production, and $\mathrm{T}$ cell adhesion [81]. Combining cancer vaccines or adoptive $\mathrm{T}$ cell transfer to immune checkpoint inhibitors, IDO inhibitors or other therapies boosting the immune system should soon represent a forefront strategy to fight cancer. 


\section{Text Box 1 - Central tolerance to tumor-specific antigens}

Central tolerance to self antigens is established in the thymus during $\mathrm{T}$ cell development, through the sequential steps of positive selection, which occurs in the thymic cortex and retains only $\mathrm{T}$ cells (thymocytes) whose $\mathrm{T}$ cell receptor (TCR) can interact with self MHC, and negative selection, which eliminates $\mathrm{T}$ cells that recognize self peptides presented in the thymic medulla. Thus, the anti-tumor $\mathrm{T}$ cell repertoire present in the periphery is shaped by the ability of the thymic medulla to process and present tumorassociated antigenic peptides. $T$ cells directed against mutated and viral antigens, which are not expressed by the thymic medulla, are not eliminated in the thymus and high affinity $\mathrm{T}$ cells to these antigens are therefore present in the periphery, probably explaining the prevalence of the spontaneous anti-tumor responses to mutated antigens $[2,12]$.

On the other hand, the presence in the blood of cancer patients of CTL directed against antigens encoded by cancer-germline genes or differentiation antigens indicates that natural immune tolerance against these antigens is either incomplete or absent. Interestingly, antigenic peptides encoded by $M A G E$ genes are usually better produced by the immuno- or the intermediate proteasomes than by the standard proteasome (Table 1) $[46,54]$. This contrasts with differentiation antigens, usually produced by the standard proteasome but not by the immuno- or the intermediate proteasomes. Because both cancer germline and differentiation genes are expressed at low level in medullary thymic epithelial cells (mTEC) [82], one would expect that $\mathrm{T}$ cells recognizing these antigens would be eliminated in the thymus. One explanation for the presence of $\mathrm{T}$ cells directed against such antigens in the blood of cancer patient could be that the cells mediating 
negative selection (the mTEC or the thymic DC) cannot efficiently process these antigens. Further study of the type of proteasome present in thymic epithelial and dendritic cells might help better understand how the circulating $\mathrm{T}$ cell repertoire is established.

\section{Text Box 2 -The proteasome and production of antigenic peptides}

The involvement of the proteasome in the production of antigenic peptides was originally stressed by the fact that presentation of the model peptide ovalbumin was dependent on ubiquitination and therefore on the $26 \mathrm{~S}$ proteasome $[83,84]$. In line with this, increasing the rate of protein ubiquitination by replacing the N-terminus with a bulky or a charged residue accelerated MHC class I presentation [85]. Recent work by Fiebiger et al., confirmed the essential role of polyubiquitination of lysine- 48 in MHC class I antigen presentation, but suggested polyubiquitination of a putative chaperone rather than the antigenic protein itself [86]. Additional evidence for the involvement of proteasome in antigen presentation is the fact that proteasome inhibitors limit the overall peptide supply to MHC class I $[87,88]$. Moreover, proteasome inhibitors were shown to inhibit the processing of specific antigenic peptides from full length proteins, while they did not affect the presentation of antigenic peptides from minigenes of the correct size (see glossary), suggesting that they indeed affect the release of peptides from full-length proteins [87]. However, the role of proteasome inhibitors is often hard to interpret, first because inhibitors generally induce ER stress, which can affect the rate of synthesis of some proteins [89], and second because they inhibit some catalytic activities of the proteasome but not others [47]. Moreover, the production of antigenic peptides often depends on a balance between productive cleavages, occurring mostly at the C-terminus, 
and destructive cleavages occurring within the sequence of the antigenic peptide $[46,55]$. Therefore, the net effect of proteasome inhibitors on peptide release depends on the relative inhibition of the catalytic activities responsible for the productive versus destructive cleavages.

\section{Text Box 3 - Transfusion of $T$ cells with affinity-enhanced TCR: use with caution}

Affinity-enhanced TCR-transduced T cells should be used with caution as they can induce deleterious, sometimes fatal, outcomes. Following transfer of $\mathrm{T}$ cells transfected with an affinity-enhanced TCR recognizing a peptide derived from MAGE-A3, some patients showed severe brain toxicity and two patients died [66]. This was due to the attack of neuronal tissue by the engineered $\mathrm{T}$ cells, which likely cross-reacted with a closely related peptide derived from MAGE-A12, whose low-level expression was recently reported in the brain. In another trial, adoptive transfer of $\mathrm{T}$ cells engineered to express an affinity-enhanced TCR directed against another MAGE-A3 peptide (EVDPIGHLY) led to a serious adverse event and fatal toxicity against the cardiac muscle due to cross-recognition of peptide ESDPIVAQY derived from the muscle protein TITIN $[67,68]$. The unmodified original TCR did not cross-recact with the TITIN peptide, indicating that affinity enhancement of the TCR was responsible for this harmful cross-reactivity leading to off-target toxicity. This is because the in vitro modified TCR no longer passes through the natural quality control filter of the thymus, whose function is to eliminate all $\mathrm{T}$ cells bearing a TCR that recognizes a self peptide. Severe on-target toxicity can also be observed if the antigen targeted has a low specificity for the tumor: harmful side effects were observed using affinity-enhanced TCR targeting 
differentiation antigens gp100, MELAN-A [64] or CEA [65], or when transferring T cells carrying a chimeric receptor against carbonic anhydrase IX [90] or against ERBB2, which happened to be lethal due to the rapid gathering of the infused $\mathrm{T}$ cells at the lung epithelium, which expresses ERBB2 at a low level [91]. 


\begin{tabular}{|c|c|c|c|c|c|c|c|}
\hline Peptide source & Peptide sequence & $\begin{array}{l}\text { MHC } \\
\text { restriction }\end{array}$ & $\begin{array}{c}\text { Standard } \\
\text { proteasome }\end{array}$ & $\begin{array}{c}\text { Intermediate } \\
\text { proteasome } \\
\text { B5i }\end{array}$ & $\begin{array}{c}\text { Intermediate } \\
\text { proteasome } \\
\text { B1i-B5i }\end{array}$ & $\begin{array}{l}\text { Immuno- } \\
\text { proteasome }\end{array}$ & $\begin{array}{l}\text { References } \\
\text { For } \\
\text { Processing }\end{array}$ \\
\hline \multicolumn{8}{|l|}{ Self } \\
\hline $\mathrm{RU}_{34-42}$ & VPYGSFKHV & HLA-B51 & $++^{\mathrm{b}}$ & n.d. & n.d. & $+/-$ & {$[53,55]$} \\
\hline FGF-5 $172-176$ and $217-220{ }^{a}$ & NTYAS_PRFK & HLA-A3 & ++ & n.d. & n.d. & - & [92] \\
\hline \multicolumn{8}{|l|}{ Differentiation } \\
\hline gp100 $40-42$ and $47-52^{a}$ & RTK_QLYPEW & HLA-A32 & ++ & n.d. & n.d. & $+/-$ & [92] \\
\hline gp100 $209-217$ & ITDQVPFSV & HLA-A2 & ++ & $+/-$ & $+/-$ & $+/-$ & {$[54,55]$} \\
\hline gp100195-202 and 191 or $192^{a}$ & RSYVPLAH_R & HLA-A3 & ++ & n.d. & n.d. & n.d. & {$[42]$} \\
\hline Tyrosinase $_{369-377}$ & YMDGTMSQV & HLA-A2 & ++ & - & $+/-$ & $+/-$ & {$[54,55]$} \\
\hline $\begin{array}{l}\text { Tyrosinase } 368-373 \text { and } 336- \\
\quad{ }^{\circ}\end{array}$ & IYMDGT_ADFSF & HLA-A24 & ++ & n.d. & n.d. & - & [92] \\
\hline Melan- $\mathrm{A}_{26-35}$ & EAAGIGILTV & HLA-A2 & ++ & $+/-$ & $+/-$ & - & {$[54]$} \\
\hline \multicolumn{8}{|l|}{ Cancer germline } \\
\hline MAGE-A3 ${ }_{114-122}$ & AELVHFLLL & HLA-B40 & - & ++ & ++ & ++ & {$[54,55,93]$} \\
\hline MAGE-A3 $271-279$ & FLWGPRALV & HLA-A2 & - & ++ & - & - & [46] \\
\hline MAGE-A $10_{254-262}$ & GLYDGMEHL & HLA-A2 & - & - & ++ & - & {$[46]$} \\
\hline MAGE-C2 $336-344$ & ALKDVEERV & HLA-A2 & - & - & ++ & ++ & {$[54,55]$} \\
\hline MAGE-C2 $2_{42-50}$ & ASSTLYLVF & HLA-B57 & - & ++ & ++ & ++ & [54] \\
\hline MAGE-C2 $2_{191-200}$ & LLFGLALIEV & HLA-A2 & - & - & ++ & - & {$[54]$} \\
\hline \multicolumn{8}{|l|}{ Mutation } \\
\hline $\mathrm{CLPP}_{240-248}$ & ILDKVLVHL & HLA-A2 & $+/-$ & n.d. & n.d. & ++ & {$[78]$} \\
\hline HAUS3 $_{154-162}$ & ILNAMIAKI & HLA-A2 & - & n.d. & ++ & n.d. & {$[18]$} \\
\hline \multicolumn{8}{|c|}{ Minor histocompatibility antigen } \\
\hline SP110 $296-301$ and $286-289^{a, c}$ & SLPRGT_STPK & HLA-A3 & $+/-$ & n.d. & n.d. & ++ & [92] \\
\hline
\end{tabular}




\section{Figure 1: Processing and presentation of different categories of tumor antigens.}

(A) MHC class I presentation pathway. HLA class I/peptide complexes recognized by cytolytic T lymphocytes (CTL) originate from the degradation of intracellular proteins by the proteasome, a large muticatalytic complex responsible for overall protein degradation. Peptides released from proteasome degradation are transported into the lumen of the endoplasmic reticulum (ER) by the transporter associated with antigen processing TAP. Peptides with extended N-terminus can be further trimmed by the ER-associated aminopeptidase (ERAAP) and then loaded on HLA class I, with the help of the peptide loading complex. Peptide/HLA complexes then migrate to the cell surface where they can be recognized by CTL. $(B, C)$ Tumor antigens are classified into two categories: Antigens with high tumor specificity (B) and antigens with low tumor specificity (C). Antigens with high tumor specificity (B) are encoded either by viral genes, mutated genes or cancer-germline genes. Viral genes are only expressed in virally infected cells. Mutations can create antigenic peptides, often called neoantigens, by modifying a peptide that already binds to the HLA molecule, or by creating a new anchor site for the HLA class I molecules. Cancer-germline genes are expressed by tumors as a result of genome-wide demethylation that also occurs in germline cells. However, germline cells lack HLA class I and therefore do not present peptides on their surface. Antigens with low tumor specificity (C) are encoded either by differentiation genes that are expressed by tumors and the corresponding healthy tissue or by genes that are overexpressed in tumor cells.

Figure 2: The Proteasome. (A) The $20 \mathrm{~S}$ proteasome is a barrel shaped complex composed of four stacked heptameric rings $\left(\alpha_{1-7}-\beta_{1-7}-\beta_{1-7}-\alpha_{1-7}\right)$, which delineate a catalytic 
chamber inside which proteins are degraded. The $20 \mathrm{~S}$ proteasome is composed of two outer $\alpha$-rings (beige) and two inner $\beta$-rings (blue). Three types of $\beta$ subunits are catalytically active (dark blue). The outer $\alpha$-rings constitute the gate of the proteasome that controls the entry of intracellular proteins inside the proteasome chamber. The $\alpha$ rings of the 20S proteasome can interact with different types of regulatory particles (B): $19 \mathrm{~S}$ regulatory particle, PA28 $\alpha \beta$, PA28 $\gamma$, PA200 and PI31. These regulatory particles can bind to one or both sides of the $20 \mathrm{~S}$ particle or form hybrid proteasomes where the $20 \mathrm{~S}$ core binds two different regulators. (C) Five proteasome subtypes exist, which differ in their catalytic subunits. The standard proteasome contains the constitutive catalytic subunits $\beta 1, \beta 2$ and $\beta 5$, while the immunoproteasome contains the interferon- $\gamma$-inducible catalytic subunits $\beta 1 \mathrm{i}, \beta 2 \mathrm{i}$ and $\beta 5 \mathrm{i}$. The intermediate proteasomes express a mixed assortment of constitutive and inducible subunits: the intermediate proteasome $\beta 5 \mathrm{i}$ contains $\beta 1, \beta 2$ and $\beta 5 i$ and the intermediate proteasome $\beta 1$ ii $\beta 5$ i contains $\beta 1 i, \beta 2$ and $\beta 5 i$. Finally, the thymoproteasome contains catalytic subunit $\beta 5$ t, which is homologous to $\beta 5$ and $\beta 5 \mathrm{i}$ and is only expressed in the cortical thymic epithelial cells (cTEC) along with $\beta 1 \mathrm{i}$ and $\beta 2 \mathrm{i}$. Each catalytic subunit has different cleavage specificities as indicated in the lower part of the figure.

\section{Figure 3: Proteasome activities}

(A) Peptide-bond hydrolysis: the hydroxyl group of the N-terminal threonine of the catalytically active subunits of the proteasome, attacks the carbonyl group of the peptide bond leading to the production of an acyl-enzyme intermediate, in which a peptide fragment remains attached to the proteasome by an ester link. Finally, a water molecule 
present in the proteasome chamber attacks the ester link between the peptide and the threonine residue, restoring the hydroxyl group of the catalytic threonine and producing the C-terminal end of the antigenic peptide.

(B) Peptide splicing by the proteasome. Here, the splicing of the antigenic peptide NTYAS_PRFK derived from the FGF-5 is shown. Following formation of the acylenzyme intermediate involving the fragment NTYAS, the free N-terminal amino-group

of peptide PRFK present in the proteasome chamber attacks the acyl-enzyme intermediate leading to the formation of the peptide NTYAS_PRFK composed of two peptide fragments originally distant in the protein.

\section{Figure 4: Priming and activation of anti-tumor $T$ cells targeting antigens processed by intermediate proteasomes and immunoproteasomes}

The initial priming of anti-tumor $\mathrm{T}$ cells takes place in secondary lymphoid tissue, such as the lymph nodes. Dendritic cells will capture tumor proteins from dying tumor cells found at the tumor site (1), then they will migrate to the sentinel lymph node (2) where peptides derived from these proteins will be cross-presented by dendritic cells on HLA class I molecules (3) to activate circulating naïve anti-tumor T cells (4). Since dendritic cells express intermediate proteasomes ( $\beta 5 \mathrm{i}$ and $\beta 1 \mathrm{i} \beta 5 \mathrm{i})$ and immunoproteasomes, antitumor $\mathrm{T}$ cells targeting antigens processed by these proteasome types should be most efficiently activated. Activated $\mathrm{T}$ cells then migrate to the tumor site (5). Because at steady state, tumors express mostly standard proteasome and intermediate proteasomes, only DC-primed T cells recognizing antigens processed by intermediate proteasomes will kill tumor cells. The IFN $\gamma$ that is produced by those $\mathrm{T}$ cells will in turn induce expression 
of the immunoproteasome by tumor cells (6). Immunoproteasome-containing tumors will then express a larger peptide repertoire containing immunoproteasome-dependent peptides, which can be targeted by other circulating $\mathrm{T}$ cells, which were also primed by DC in the lymph nodes. Because $\mathrm{T}$ cells recognizing standard proteasome-dependent antigens, such as melanoma differentiation antigens (Table 1), can be found in the blood of cancer patients, it is likely that the priming of these $\mathrm{T}$ cells is performed by the tumor cells themselves.

\section{Aknowledgments}

We are grateful to Mrs Auriane Sibille for her precious help in the preparation of this manuscript. JAH is supported by a fellowship from the Fonds National de la Recherche Scientifique (TELEVIE grant No. 7455115F).

\section{Resources}

${ }^{\mathrm{i}}$ https://www.cancerresearch.org/scientists/events-and-resources/peptide-database

\section{References}

1. Vigneron, N. and Van den Eynde, B.J. (2011) Insights into the processing of MHC class I ligands gained from the study of human tumor epitopes. Cell. Mol. Life Sci. 68 (9), 1503-1520.

2. Coulie, P.G. et al. (2014) Tumour antigens recognized by T lymphocytes: at the core of cancer immunotherapy. Nat. Rev. Cancer 14 (2), 135-146.

3. Vigneron, N. (2015) Human Tumor Antigens and Cancer Immunotherapy. Biomed. Res. Int. 2015, 948501.

4. Wolchok, J.D. et al. (2010) Ipilimumab monotherapy in patients with pretreated advanced melanoma: a randomised, double-blind, multicentre, phase 2 , dose-ranging study. Lancet Oncol. 11 (2), 155-64.

5. Topalian, S.L. et al. (2012) Safety, activity, and immune correlates of anti-PD-1 antibody in cancer. N. Engl. J. Med. 366 (26), 2443-54.

6. Vigneron, N. et al. (2013) Database of T cell-defined human tumor antigens: the 2013 update. Cancer Immun. 13, 15.

7. Iacovides, D. et al. (2013) Shared mechanisms in stemness and carcinogenesis: lessons from oncogenic viruses. Front. Cell. Infect. Microbiol. 3, 66. 
8. Welters, M.J. et al. (2008) Induction of tumor-specific CD4+ and CD8+ T-cell immunity in cervical cancer patients by a human papillomavirus type 16 E6 and E7 long peptides vaccine. Clin. Cancer Res. 14 (1), 178-87.

9. Kenter, G.G. et al. (2009) Vaccination against HPV-16 oncoproteins for vulvar intraepithelial neoplasia. N. Engl. J. Med. 361 (19), 1838-1847.

10. van Poelgeest, M.I. et al. (2013) HPV16 synthetic long peptide (HPV16-SLP) vaccination therapy of patients with advanced or recurrent HPV16-induced gynecological carcinoma, a phase II trial. J. Transl. Med. 11, 88.

11. Welters, M.J. et al. (2016) Vaccination during myeloid cell depletion by cancer chemotherapy fosters robust T cell responses. Sci. Transl. Med. 8 (334), 334-352.

12. Lennerz, V. et al. (2005) The response of autologous T cells to a human melanoma is dominated by mutated neoantigens. Proc. Natl. Acad. Sci. U.S.A. 102 (44), 16013-16018. 13. Van Allen, E.M. et al. (2015) Genomic correlates of response to CTLA-4 blockade in metastatic melanoma. Science 350 (6257), 207-211.

14. Rizvi, N.A. et al. (2015) Cancer immunology. Mutational landscape determines sensitivity to PD-1 blockade in non-small cell lung cancer. Science 348 (6230), 124-128. 15. Le, D.T. et al. (2015) PD-1 Blockade in Tumors with Mismatch-Repair Deficiency. N. Engl. J. Med. 372 (26), 2509-2520.

16. Anagnostou, V. et al. (2017) Evolution of Neoantigen Landscape during Immune Checkpoint Blockade in Non-Small Cell Lung Cancer. Cancer Discov. 7 (3), 264-276.

17. van Rooij, N. et al. (2013) Tumor exome analysis reveals neoantigen-specific T-cell reactivity in an ipilimumab-responsive melanoma. J. Clin. Oncol. 31 (32), e439-42. 18. Robbins, P.F. et al. (2013) Mining exomic sequencing data to identify mutated antigens recognized by adoptively transferred tumor-reactive T cells. Nat. Med. 19 (6), 747-752.

19. Cohen, C.J. et al. (2015) Isolation of neoantigen-specific T cells from tumor and peripheral lymphocytes. J. Clin. Invest. 125 (10), 3981-3991.

20. Parkhurst, M. et al. (2016) Isolation of T-Cell Receptors Specifically Reactive with Mutated Tumor-Associated Antigens from Tumor-Infiltrating Lymphocytes Based on CD137 Expression. Clin. Cancer Res. DOI: 10.1158/1078-0432.CCR-16-2680.

21. Kalaora, S. et al. (2016) Use of HLA peptidomics and whole exome sequencing to identify human immunogenic neo-antigens. Oncotarget 7 (5), 5110-5117.

22. Prickett, T.D. et al. (2016) Durable Complete Response from Metastatic Melanoma after Transfer of Autologous T Cells Recognizing 10 Mutated Tumor Antigens. Cancer Immunol. Res. 4 (8), 669-678.

23. Stevanovic, S. et al. (2017) Landscape of immunogenic tumor antigens in successful immunotherapy of virally induced epithelial cancer. Science 356 (6334), 200-205. 24. Rosenberg, S.A. and White, D.E. (1996) Vitiligo in patient with melanoma: normal tissue antigens can be target for cancer immunotherapy. J. Immunother. 19, 81-84. 25. Hammarstrom, S. (1999) The carcinoembryonic antigen (CEA) family: structures, suggested functions and expression in normal and malignant tissues. Semin. Cancer Biol. $9(2), 67-81$.

26. Groll, M. et al. (1997) Structure of 20S proteasome from yeast at $2.4 \AA$ resolution. Nature 386, 463-471.

27. Rabl, J. et al. (2008) Mechanism of gate opening in the $20 \mathrm{~S}$ proteasome by the proteasomal ATPases. Mol. Cell 30 (3), 360-368. 
28. Vigneron, N. and Van den Eynde, B.J. (2014) Proteasome Subtypes and Regulators in the Processing of Antigenic Peptides Presented by Class I Molecules of the Major Histocompatibility Complex. Biomolecules 4 (4), 994-1025.

29. Chu-Ping, M. et al. (1992) Purification and characterization of a protein inhibitor of the 20S proteasome (macropain). Biochim. Biophys. Acta 1119 (3), 303-311.

30. Bader, M. et al. (2011) A conserved F box regulatory complex controls proteasome activity in Drosophila. Cell 145 (3), 371-382.

31. Li, X. et al. (2014) Molecular and cellular roles of PI31 (PSMF1) protein in regulation of proteasome function. J. Biol. Chem. 289 (25), 17392-17405.

32. Sun, Y. et al. (2002) Expression of the proteasome activator PA28 rescues the presentation of a cytotoxic T lymphocyte epitope on melanoma cells. Cancer. Res. 62 (10), 2875-82.

33. Keller, M. et al. (2015) The proteasome immunosubunits, PA28 and ERaminopeptidase 1 protect melanoma cells from efficient MART-126-35 -specific T-cell recognition. Eur. J. Immunol. 45 (12), 3257-3268.

34. Löwe, J. et al. (1995) Crystal structure of the $20 \mathrm{~S}$ proteasome from the archaeon $T$. acidophilum at 3.4 Å resolution. Science 268, 533-539.

35. Seemuller, E. et al. (1995) Proteasome from Thermoplasma acidophilum: a threonine protease. Science 268 (5210), 579-582.

36. Huber, E.M. et al. (2012) Immuno- and constitutive proteasome crystal structures reveal differences in substrate and inhibitor specificity. Cell 148 (4), 727-38.

37. Vigneron, N. et al. (2004) An antigenic peptide produced by peptide splicing in the proteasome. Science 304, 587-590.

38. Hanada, K. et al. (2004) Immune recognition of a human renal cancer antigen through post-translational protein splicing. Nature 427 (6971), 252-256.

39. Warren, E.H. et al. (2006) An antigen produced by splicing of noncontiguous peptides in the reverse order. Science 313 (5792), 1444-1447.

40. Dalet, A. et al. (2010) Splicing of distant peptide fragments occurs in the proteasome by transpeptidation and produces the spliced antigenic peptide derived from fibroblast growth factor-5. J. Immunol. 184 (6), 3016-3024.

41. Dalet, A. et al. (2011) An antigenic peptide produced by reverse splicing and double asparagine deamidation. Proc. Natl. Acad. Sci. USA 108 (29), E323-331.

42. Michaux, A. et al. (2014) A Spliced Antigenic Peptide Comprising a Single Spliced Amino Acid Is Produced in the Proteasome by Reverse Splicing of a Longer Peptide Fragment followed by Trimming. J. Immunol. 192 (4), 1962-1971.

43. Ebstein, F. et al. (2016) Proteasomes generate spliced epitopes by two different mechanisms and as efficiently as non-spliced epitopes. Sci. Rep. 6, 24032.

44. Mishto, M. et al. (2012) Driving forces of proteasome-catalyzed peptide splicing in yeast and humans. Mol. Cell. Proteomics 11 (10), 1008-1023.

45. Liepe, J. et al. (2016) A large fraction of HLA class I ligands are proteasomegenerated spliced peptides. Science 354 (6310), 354-358.

46. Guillaume, B. et al. (2010) Two abundant proteasome subtypes that uniquely process some antigens presented by HLA class I molecules. Proc. Natl. Acad. Sci. U.S.A. 107 (43), 18599-18604.

47. Vigneron, N. et al. (2015) The capture proteasome assay: A method to measure proteasome activity in vitro. Anal. Biochem. 482, 7-15. 
48. Sijts, A.J.A.M. et al. (2000) Efficient generation of a hepatitis B virus cytotoxic T lymphocyte epitope requires the structural features of immunoproteasomes. J. Exp. Med. 191, 503-513.

49. Gaczynska, M. et al. (1993) $\gamma$-interferon and expression of MHC genes regulate peptide hydrolysis by proteasomes. Nature 365, 264-267.

50. Driscoll, J. et al. (1993) MHC-linked LMP gene products specifically alter peptidase activities of the proteasome. Nature 365, 262-264.

51. Kincaid, E.Z. et al. (2012) Mice completely lacking immunoproteasomes show major changes in antigen presentation. Nat. Immunol. 13 (2), 129-35.

52. de Verteuil, D. et al. (2010) Deletion of immunoproteasome subunits imprints on the transcriptome and has a broad impact on peptides presented by major histocompatibility complex I molecules. Mol. Cell. Proteomics 9 (9), 2034-47.

53. Morel, S. et al. (2000) Processing of some antigens by the standard proteasome but not by the immunoproteasome results in poor presentation by dendritic cells. Immunity $12,107-117$.

54. Guillaume, B. et al. (2012) Analysis of the processing of seven human tumor antigens by intermediate proteasomes. J. Immunol. 189 (7), 3538-3547.

55. Chapiro, J. et al. (2006) Destructive cleavage of antigenic peptides either by the immunoproteasome or by the standard proteasome results in differential antigen presentation. J. Immunol. 176, 1053-1061.

56. Tripathi, S.C. et al. (2016) Immunoproteasome deficiency is a feature of non-small cell lung cancer with a mesenchymal phenotype and is associated with a poor outcome. Proc. Natl. Acad. Sci. U.S.A. 113 (11), E1555-1564.

57. Woods, K. et al. (2016) Mismatch in epitope specificities between IFNgamma inflamed and uninflamed conditions leads to escape from $\mathrm{T}$ lymphocyte killing in melanoma. J. Immunother. Cancer 4, 10.

58. Rouette, A. et al. (2016) Expression of immunoproteasome genes is regulated by cellintrinsic and -extrinsic factors in human cancers. Sci. Rep. 6, 34019.

59. Dahlmann, B. et al. (2000) Different proteasome subtypes in a single tissue exhibit different enzymatic properties. J. Mol. Biol. 303, 643-653.

60. Murata, S. et al. (2007) Regulation of CD8+ T cell development by thymus-specific proteasomes. Science 316 (5829), 1349-1353.

61. Nitta, T. et al. (2010) Thymoproteasome shapes immunocompetent repertoire of CD8+ T cells. Immunity 32 (1), 29-40.

62. Gattinoni, L. et al. (2006) Adoptive immunotherapy for cancer: building on success. Nat. Rev. Immunol. 6 (5), 383-393.

63. Khammari, A. et al. (2007) Long-term follow-up of patients treated by adoptive transfer of melanoma tumor-infiltrating lymphocytes as adjuvant therapy for stage III melanoma. Cancer Immunol. Immunother. 56 (11), 1853-1860.

64. Johnson, L.A. et al. (2009) Gene therapy with human and mouse T-cell receptors mediates cancer regression and targets normal tissues expressing cognate antigen. Blood 114 (3), 535-46.

65. Parkhurst, M.R. et al. (2011) T cells targeting carcinoembryonic antigen can mediate regression of metastatic colorectal cancer but induce severe transient colitis. Mol. Ther. $19(3), 620-6$. 
66. Morgan, R.A. et al. (2013) Cancer regression and neurological toxicity following anti-MAGE-A3 TCR gene therapy. J. Immunother. 36 (2), 133-51.

67. Cameron, B.J. et al. (2013) Identification of a Titin-derived HLA-A1-presented peptide as a cross-reactive target for engineered MAGE A3-directed T cells. Sci. Transl. Med. 5 (197), 197ra103.

68. Linette, G.P. et al. (2013) Cardiovascular toxicity and titin cross-reactivity of affinityenhanced T cells in myeloma and melanoma. Blood 122 (6), 863-71.

69. Rosenberg, S.A. et al. (1988) Use of tumor-infiltrating lymphocytes and interleukin-2 in the immunotherapy of patients with metastatic melanoma. N. Engl. J. Med. 319, 16761680.

70. Gross, S. et al. (2017) Twelve-year survival and immune correlates in dendritic cellvaccinated melanoma patients. JCI Insight 2 (8).

71. Naslund, T.I. et al. (2007) Comparative prime-boost vaccinations using Semliki Forest virus, adenovirus, and ALVAC vectors demonstrate differences in the generation of a protective central memory CTL response against the P815 tumor. J. Immunol. 178 (11), 6761-6769.

72. Chapatte, L. et al. (2006) Processing of tumor-associated antigen by the proteasomes of dendritic cells controls in vivo T-cell responses. Cancer Res. 66, 5461-5468.

73. Dannull, J. et al. (2013) Melanoma immunotherapy using mature DCs expressing the constitutive proteasome. J. Clin. Invest. 123 (7), 3135-3145.

74. Muchamuel, T. et al. (2009) A selective inhibitor of the immunoproteasome subunit LMP7 blocks cytokine production and attenuates progression of experimental arthritis.

Nat. Med. 15 (7), 781-787.

75. Ho, Y.K. et al. (2007) LMP2-specific inhibitors: chemical genetic tools for proteasome biology. Chem. Biol. 14 (4), 419-430.

76. Kuhn, D.J. et al. (2009) Targeted inhibition of the immunoproteasome is a potent strategy against models of multiple myeloma that overcomes resistance to conventional drugs and nonspecific proteasome inhibitors. Blood 113 (19), 4667-4676.

77. Calis, J.J. et al. (2015) Role of peptide processing predictions in T cell epitope identification: contribution of different prediction programs. Immunogenetics 67 (2), 8593.

78. Corbiere, V. et al. (2011) Antigen spreading contributes to MAGE vaccinationinduced regression of melanoma metastases. Cancer Res. 71 (4), 1253-62.

79. Obeng, E.A. et al. (2006) Proteasome inhibitors induce a terminal unfolded protein response in multiple myeloma cells. Blood 107 (12), 4907-4916.

80. Platten, M. et al. (2012) Tryptophan catabolism in cancer: beyond IDO and tryptophan depletion. Cancer. Res. 72 (21), 5435-40.

81. Pedoeem, A. et al. (2014) Programmed death-1 pathway in cancer and autoimmunity. Clin. Immunol. 153 (1), 145-52.

82. Gotter, J. et al. (2004) Medullary epithelial cells of the human thymus express a highly diverse selection of tissue-specific genes colocalized in chromosomal clusters. $J$. Exp. Med. 199, 155-166.

83. Michalek, M.T. et al. (1993) A role for the ubiquitin-dependent proteolytic pathway in MHC class I-restricted antigen presentation. Nature 363 (6429), 552-4. 
84. Ben-Shahar, S. et al. (1997) Production of a specific major histocompatibility complex class I-restricted epitope by ubiquitin-dependent degradation of modified ovalbumin in lymphocyte lysate. J. Biol. Chem. 272 (34), 21060-6.

85. Grant, E.P. et al. (1995) Rate of antigen degradation by the ubiquitin-proteasome pathway influences MHC class I presentation. J. Immunol. 155 (8), 3750-8.

86. Fiebiger, B.M. et al. (2015) Polyubiquitination of lysine- 48 is an essential but indirect signal for MHC class I antigen processing. Eur. J. Immunol. 45 (3), 716-727.

87. Rock, K.L. et al. (1994) Inhibitors of the proteasome block the degradation of most cell proteins and the generation of peptides presented on MHC class I molecules. Cell 78, 761-771.

88. Cerundolo, V. et al. (1997) The proteasome-specific inhibitor lactacystin blocks presentation of cytotoxic T lymphocyte epitopes in human and murine cells. Eur. J. Immunol. 27, 336-341.

89. Nawrocki, S.T. et al. (2005) Bortezomib inhibits PKR-like endoplasmic reticulum (ER) kinase and induces apoptosis via ER stress in human pancreatic cancer cells. Cancer. Res. 65 (24), 11510-9.

90. Lamers, C.H. et al. (2006) Treatment of metastatic renal cell carcinoma with autologous T-lymphocytes genetically retargeted against carbonic anhydrase IX: first clinical experience. J. Clin. Oncol. 24 (13), e20-22.

91. Morgan, R.A. et al. (2010) Case report of a serious adverse event following the administration of $\mathrm{T}$ cells transduced with a chimeric antigen receptor recognizing ERBB2. Mol. Ther. 18 (4), 843-51.

92. Dalet, A. et al. (2011) Differences in the production of spliced antigenic peptides by the standard proteasome and the immunoproteasome. Eur. J. Immunol. 41 (1), 39-46. 93. Schultz, E.S. et al. (2002) The production of a new MAGE-3 peptide presented to cytolytic T lymphocytes by HLA-B40 requires the immunoproteasome. J. Exp. Med. 195, 391-399. 


\section{Glossary}

Adoptive T-cell therapy: in cancer immunotherapy, adoptive T-cell therapy consists in infusing patients with autologous T cells that have been amplified in vitro. These $\mathrm{T}$ cells can either be native T cells isolated directly from the blood or the tumor of a patient, or T cells transduced to express a specific TCR or chimeric receptor.

Myeloid-derived suppressor cells (MDSC): heterogeneous group of cells originating from the myeloid lineage and displaying strong immunosuppressive properties thereby regulating the function of $\mathrm{T}$ cells, dendritic cells, natural killer cells or macrophages. Although in mice, specific markers can be used to characterize these cells (CD11b ${ }^{+}$ $\mathrm{GR} 1^{+}$), phenotypic characterization of human MDSC is still not well established: human MDSC are generally defined as $\mathrm{CD}_{3} 3^{+}, \mathrm{C}_{11 \mathrm{~b}^{+}}$and $\mathrm{HLA}-\mathrm{DR}{ }^{\text {low }}$ myeloid cells. Immunodominant response: immunological response, which mainly targets a few antigenic peptides among many others.

Genome-wide demethylation : Cytosines in CpG sites (region of the DNA where a cytosine nucleotide is followed by a guanine nucleotide) are often methylated in the mammalian genome. This epigenetic mark is usually associated with transcriptional silencing. In tumors and in germline cells, a genome-wide demethylation takes place that unleashes expression of genes such as those encoding the MAGE-type proteins. Aminopeptidases: proteolytic enzymes that catalyze the cleavage of amino acids from the amino terminus of proteins or peptides.

Immunopeptidome/peptide repertoire: set of peptides that are associated to the HLA class I molecules expressed by a given cell type. To analyze the immunopeptidome, HLA-peptide complexes are immunoprecipitated using specific antibodies. Peptides 
bound to the purified HLA molecules are then eluted using an acidic buffer, and the eluted peptide mixture is then analyzed by mass spectrometry.

Minigene construct: DNA construct that leads to the expression of the antigenic peptide already processed to the final size allowing its loading onto the HLA class I molecule. This form of antigen does not need intracellular processing by the proteasome or other proteases. 


\section{Tumor site}

1- Uptake of proteins bearing tumor antigen by dendritic cells
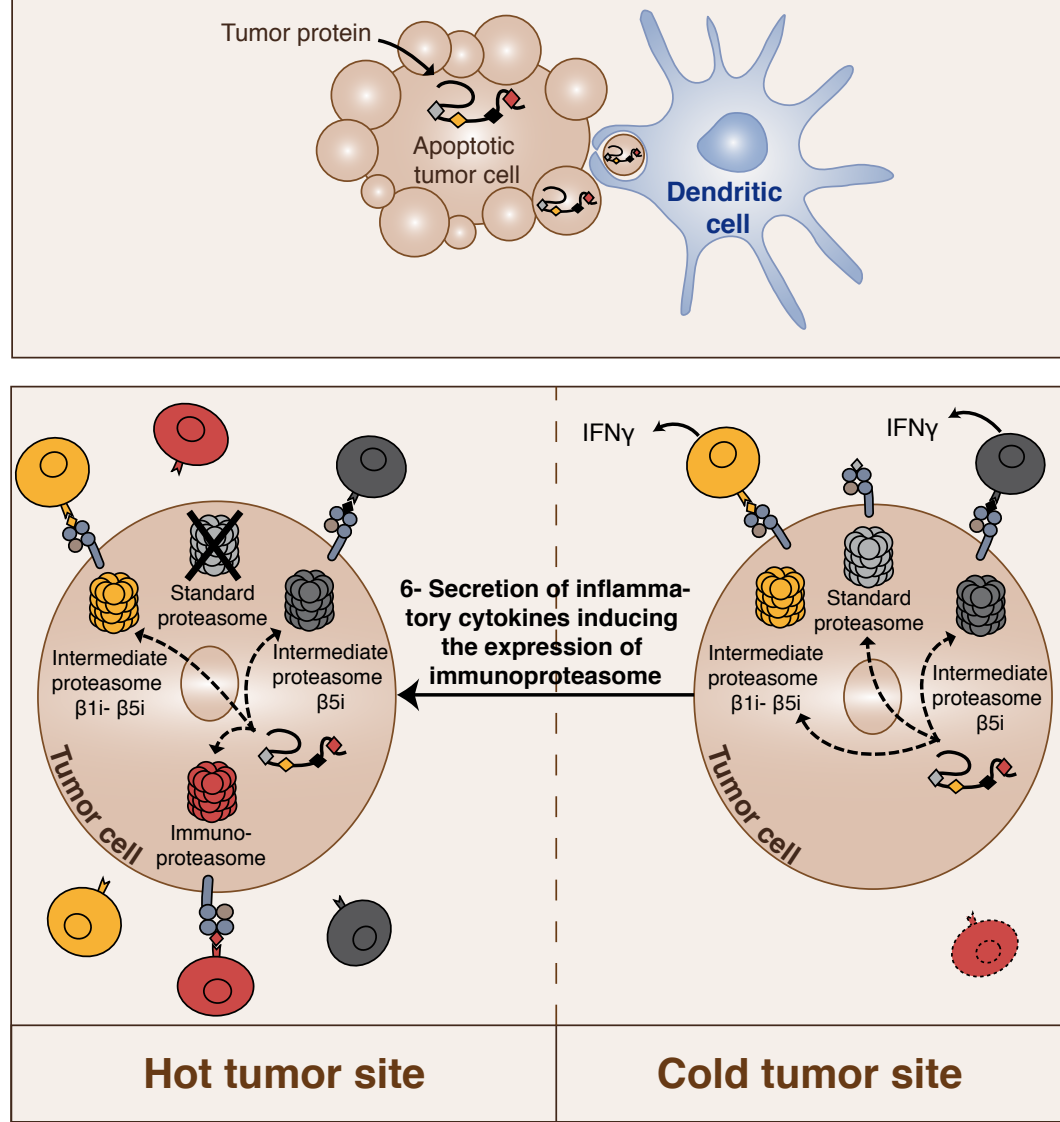

\section{Sentinel lymph node}

\section{2- Migration of the dendritic cell to}

the lymph node
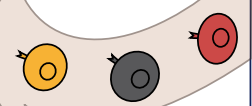

5- Migration of primed anti-tumor $\mathrm{T}$ cells to tumor site
3- Proteasome -dependent

cross-presentation

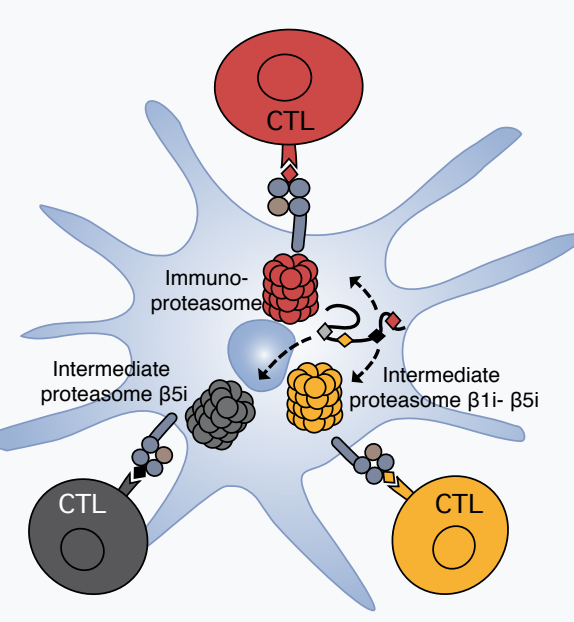

4- Priming of naïve anti-tumor $T$ cells 


\section{A Hydrolysis}

MAGE-A3 amino acid sequence:

MPLEQRSQHCKPEEGLEARGEALGLVGAQAPA TEEQEAASSSSTLVEVTLGEVPAAESPDPPQSP QGASSLPTTMNYPLWSQSYEDSSNQEEEGPST FPDLESEFQAALSRKVAELVHFLLLKYRAREPVT KAEMLGSVVGNWQYFFPVIFSKASSSLQLVFGIE LMEVDPIGHLYIFATCLGLSYDGLLGDNQIMPKA GLLIIVLAIIAREGDCAPEEKIWEELSVLEVFEGRE DSILGDPKKLLTQHFVQENYLEYRQVPGSDPAC YEFLWGPRALVETSYVKVLHHMVKISGGPHISYP PLHEWVLREGEE

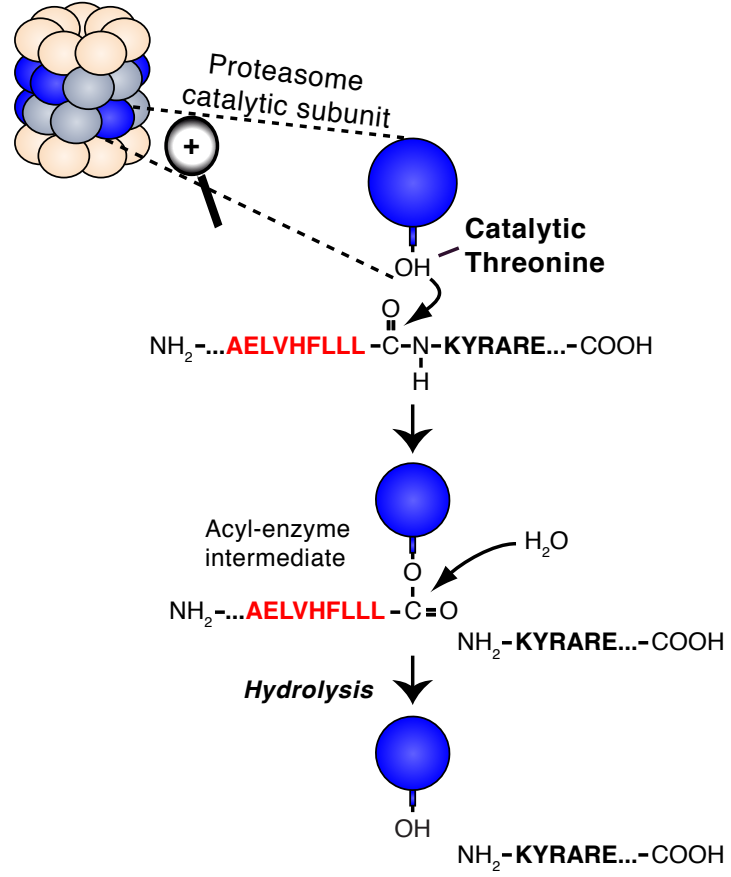

$\mathrm{NH}_{2}-\ldots$ AELVHFLLL-COOH

\section{B Splicing}

FGF5 amino acid sequence:

MSLSFLLLLFFSHLILSAWAHGEKRLAPKGQPGP AATDRNPRGSSSRQSSSSAMSSSSASSSPAASL GSQGSGLEQSSFQWSPSGRRTGSLYCRVGIGF HLQIYPDGKVNGSHEANMLSVLEIFAVSQGIVGIR GVFSNKFLAMSKKGKLHASAKFTDDCKFRERFQ ENSYNTYASAIHRTEKTGREWYVALNKRGKAKR GCSPRVKPQHISTHFLPRFKQSEQPELSFTVTVP EKKKPPSPIKPKIPLSAPRKNTNSVKYRLKFRFG

Proteasome catalytic subunits

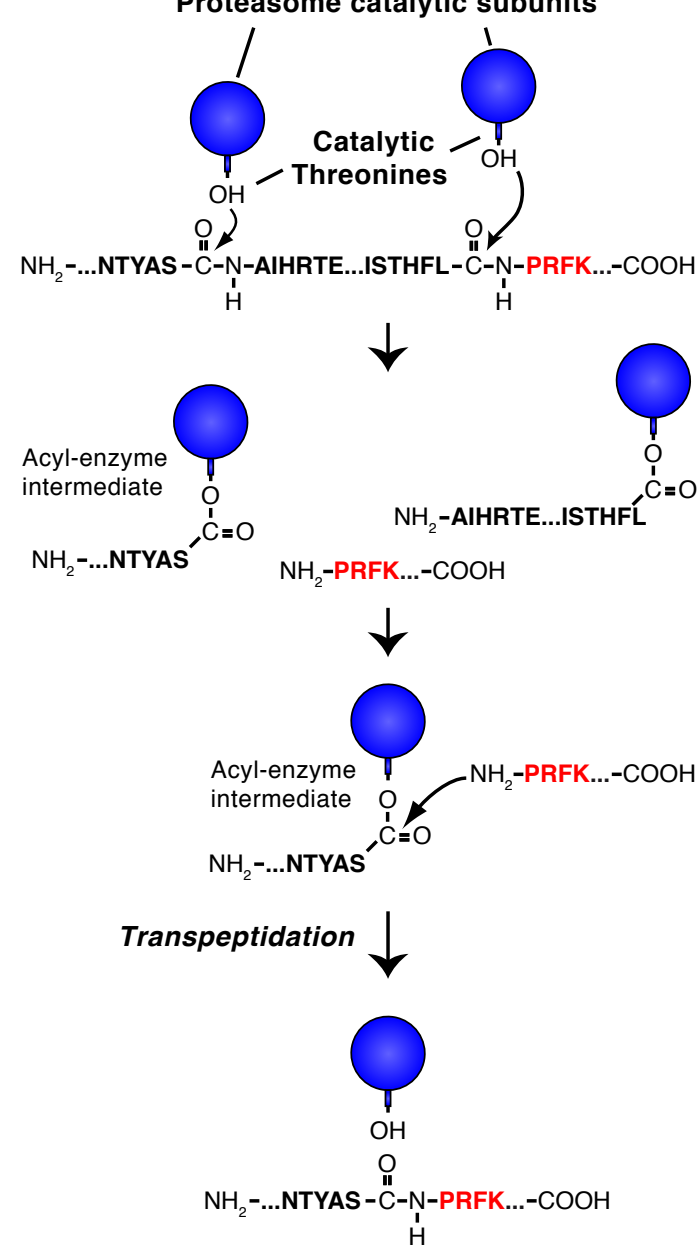




\section{A The 20S proteasome}

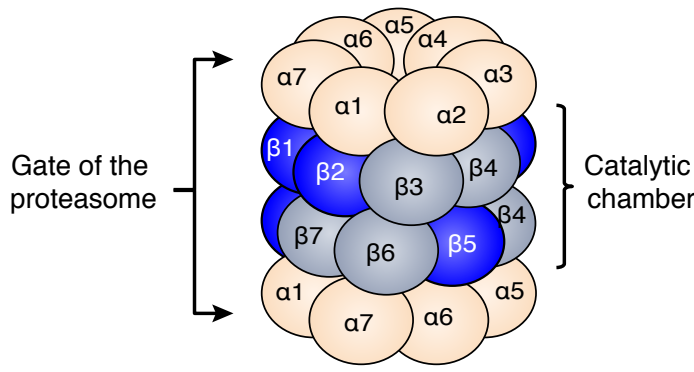

\section{Proteasome subtypes}

\section{Standard} proteasome

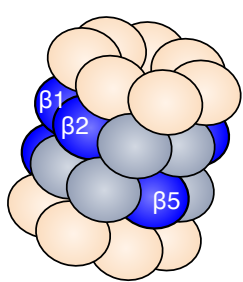

$\beta 1-\beta 2-\beta 5$

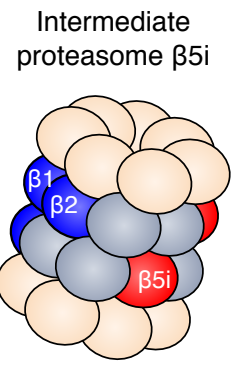

$\beta 1-\beta 2-\beta 5 i$
Intermediate proteasome $\beta 1 \mathrm{i}-\beta 5 \mathrm{i}$

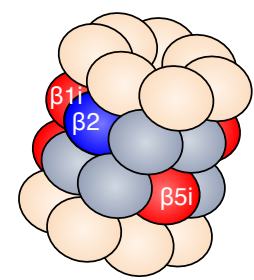

$\beta 1 \mathrm{i}-\beta 2-\beta 5 \mathrm{i}$
B Regulatory Particles

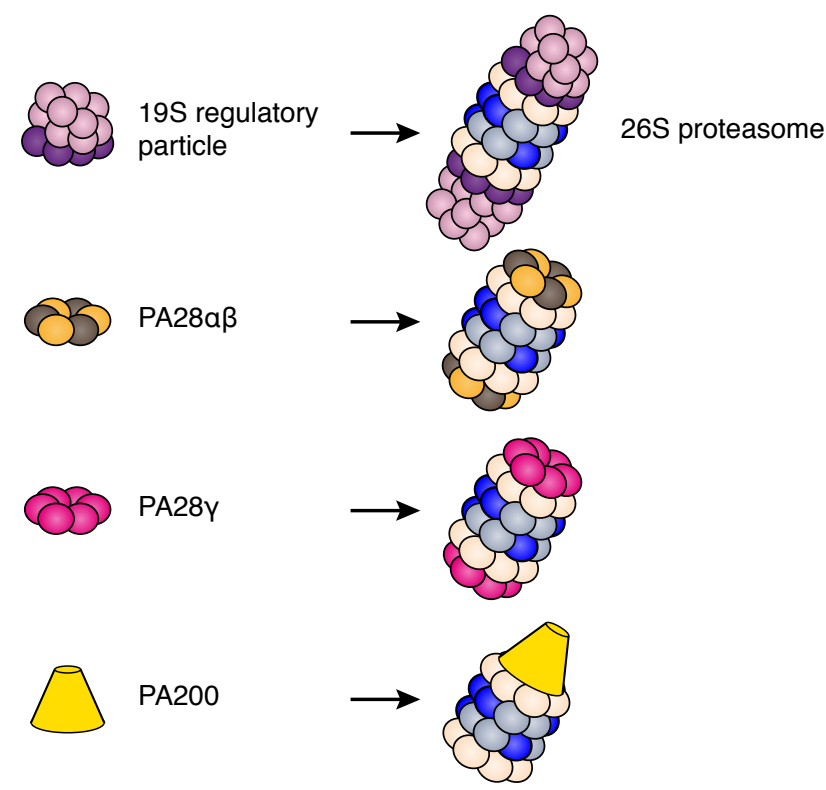

PI31

$\beta 1$ : PGPH or caspase-like activity

$\beta 2$ : Trypsin-like activity

$\beta 5$ : Chymotrypsin-like activity

Immunoproteasome

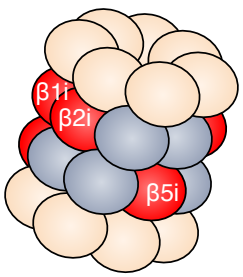

$\beta 1 \mathrm{i}-\beta 2 \mathrm{i}-\beta 5 \mathrm{i}$

$\beta 1$ i: Chymotrypsin or BrAAP like activity

B2i: Trypsin-like activity

$\beta 5 \mathrm{i}$ : Chymotrypsin-like activity
Thymoproteasome

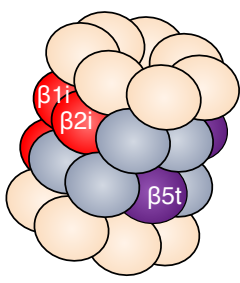

$\beta 1 \mathrm{i}-\beta 2 \mathrm{i}-\beta 5 \mathrm{t}$

ß5t: Reduced chymotrypsin-like activity 


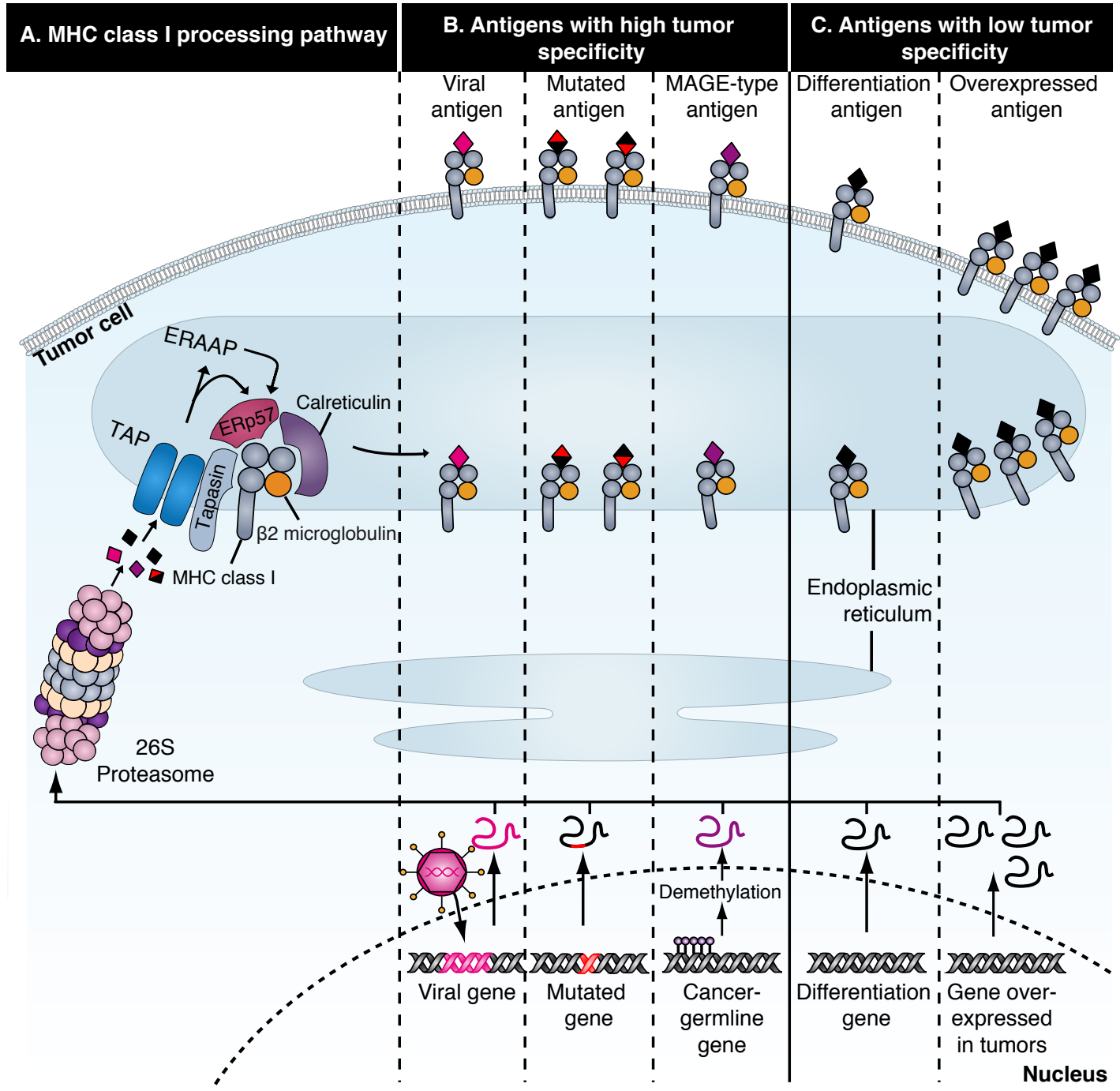

